\title{
Nouvelles interactions numériques et apprentissages scolaires : Entretien avec Mireille Bétrancourt
}

\author{
New digital interactions and school learning: \\ Interview with Mireille Bétrancourt
}

\section{Nuevas interacciones digitales y aprendizaje escolar: Entrevista con Mireille Bétrancourt}

Stéphanie Fleck, maître de conférences

Université de Lorraine, France

stephanie.fleck@univ-lorraine.fr

Luc Massou, maître de conférences

Université de Lorraine, France

luc.massou@univ-lorraine.fr

\begin{abstract}
RÉSUMÉ
Mireille Bétrancourt est professeure en technologies de l'information et processus d'apprentissage à la Faculté de psychologie et des sciences de l'éducation de l'Université de Genève (Suisse), où elle dirige l'unité de Technologies de formation et d'apprentissage (TECFA). L'objet général de ses travaux porte sur la conception des ressources numériques pédagogiques dans une perspective cognitive et ergonomique, et sur les usages des technologies numériques dans différents contextes de formation et d'enseignement. Dans cet entretien, elle souligne l'importance de la congruence technopédagogique des nouvelles interactions sensorimotrices avec les exigences de la tâche d'apprentissage et du contexte scolaire dans lequel elles sont utilisées afin de mieux en évaluer les apports réels.
\end{abstract}

Mots-clés: interactions sensorimotrices, multimodalité sensorielle, tâche d'apprentissage, congruence technopédagogique 
Mireille Bétrancourt is a professor of Information Technology and Learning Processes at the Faculty of Psychology and Educational Sciences of the University of Geneva (Switzerland), where she heads the Training and Learning Technologies Unit (TECFA). Her work's general focus is on designing digital educational resources from a cognitive and ergonomic perspective and the uses of digital technologies in different training and teaching contexts. In this interview, she underlines the importance of techno-pedagogical congruence of new sensory-motor interactions with the learning task's requirements and the school context in which they are used to evaluate their real contributions better.

Keywords: sensory-motor interactions, sensory multimodality, learning task, technopedagogical congruence

\section{RESUMEN}

Mireille Bétrancourt es profesora de Tecnología de la Información y Procesos de Aprendizaje en la Facultad de Psicología y Ciencias de la Educación de la Universidad de Ginebra (Suiza), donde dirige la Unidad de Tecnologías de la Formación y el Aprendizaje (TECFA). El enfoque general de su trabajo se centra en el diseño de recursos educativos digitales desde una perspectiva cognitiva y ergonómica, y en los usos de las tecnologías digitales en diferentes contextos de capacitación y de enseñanza. En esta entrevista, subraya la importancia de la congruencia tecno-pedagógica entre las nuevas interacciones sensoriales-motoras y los requisitos de la tarea de aprendizaje y el contexto escolar en el que se utilizan, con el objetivo de evaluar mejor sus contribuciones reales.

Palabras clave: interacciones sensoriales-motoras, multimodalidad sensorial, tarea de aprendizaje, congruencia tecnopedagógica

Mireille Bétrancourt est titulaire d'un doctorat en sciences cognitives obtenu à l'Institut national polytechnique de Grenoble en 1996, suivi de plusieurs expériences postdoctorales, notamment à l'Université de Stanford aux États-Unis. Elle rejoint l'Université de Genève en 2003 en tant que professeure en technologies de l'information et processus d'apprentissage à la Faculté de psychologie et des sciences de l'éducation. Elle dirige l'unité de Technologies de formation et d'apprentissage (TECFA), qui coordonne une maitrise spécialisée, le Master MALTT, dans le domaine des technologies de l'éducation.

Ses domaines d'expertise portent sur deux axes. Le premier axe concerne la conception des ressources numériques pédagogiques (multimédia, logiciels éducatifs, jeu sérieux) dans une perspective cognitive et ergonomique. Le deuxième axe explore les usages des technologies numériques dans différents contextes de formation et d'enseignement, en documentant leurs effets (perçus et observables) et leur appropriation par les acteurs. L'objectif de ses recherches est de contribuer à enrichir les connaissances sur les processus d'apprentissage et d'enseignement ayant recours aux technologies numériques ainsi que de fournir des recommandations issues de la recherche pour la conception des dispositifs médiatisés. 
Nous remercions Mireille Bétrancourt pour l'entretien qu'elle nous a accordé pour ce numéro thématique de la revue Médiations et médiatisations sur les nouvelles interfaces et interactions avec le numérique pour l'apprentissage collaboratif, pour lequel elle souligne en particulier l'importance de la congruence technopédagogique des nouvelles interactions sensorimotrices avec les exigences de la tâche d'apprentissage et du contexte scolaire dans lequel elles sont utilisées afin de mieux en évaluer les apports réels.

STÉPHANIE FLECK et LUC MASSOU : Vous avez contribué dans vos recherches à la conception de ressources numériques pédagogiques dans une perspective à la fois cognitive et ergonomique. Comment qualifierez-vous l'impact des interfaces numériques de nouvelle génération (tactiles, tangibles, objets connectés...) sur les apprentissages collaboratifs en contexte scolaire?

MIREILLE BÉTRANCOURT: Comme pour toute technologie éducative, je pense qu'il est difficile de dissocier les effets des outils des conditions de leur mise en œuvre. Dans les cas des interfaces numériques, on peut considérer schématiquement trois dimensions : le contexte d'enseignement (par exemple en classe ou chez soi, en situation individuelle ou collaborative), le contenu et la forme de la tâche d'apprentissage et, en troisième lieu, les caractéristiques de l'outil. De nombreux modèles comme le TPACK (Mishra et Keller, 2006) ou la théorie instrumentale de Rabardel (1995) pointent la nécessaire congruence entre les caractéristiques de l'outil, la situation d'enseignement et les exigences de la tâche d'apprentissage. Si bien qu'il est difficile d'isoler l'impact des outils sans prendre en considération la situation dans laquelle ils sont utilisés et pour quel objectif. Cela étant dit, une caractéristique importante des interfaces citées est le fait qu'elles font appel à la sensorimotricité, ce qui ajoute l'apport du geste et du toucher au traitement symbolique des informations. De nombreuses études montrent l'apport du geste et de la multimodalité sensorielle pour les apprentissages symboliques, notamment dans le cadre théorique de la cognition incarnée (Goldin-Meadow, 2014). Cette caractéristique peut être considérée comme une affordance facilitant l'apprentissage si elle est congruente avec les exigences de la tâche : pour prendre l'exemple des tablettes tactiles, cette caractéristique est plus utile pour apprendre à tracer des lettres que pour lire un texte non interactif (Amadieu, Mulet, Van der Linden, Lombard et Van de Leemput, sous presse). Dans une situation collaborative, je considère que cette caractéristique sensorimotrice devrait soutenir les interactions entre les élèves en rendant visibles, en vérifiant les hypothèses de chacun. La réification du travail des élèves peut également soutenir la régulation et l'orchestration par les enseignants (Dillenbourg et Jermann, 2010).

STÉPHANIE FLECK et LUC MASSOU : Selon vous, quelles formes d'interactions entre apprenants, et/ou entre apprenants et enseignants, l'ergonomie des systèmes hypermédias qui ne se fondent pas sur l'utilisation des claviers/écrans/souris favorise-t-elle?

MIREILLE BÉTRANCOURT : Je définirais ici l'ergonomie des systèmes hypermédias sur la base de leur facilité d'apprentissage et d'utilisation, leur soutien à l'atteinte des tâches (utilité) et leur acceptabilité par les différents acteurs (Bétrancourt, 2019). En termes de facilité d'utilisation, les systèmes ergonomiques sans clavier ni souris devraient limiter l'effort cognitif non utile, parce que la manipulation des objets de la tâche est plus directe (Hutchins, Hollan et Norman, 1986). En effet, dans ces interfaces on agit directement sur les objets de la tâche avec ses mains, ce qui permet également de réintroduire la coordination œilmain ou boucle perceptivo-motrice qui est perdue lorsque la manipulation est médiatisée par le clavier ou la souris (Bara et Gentaz, 2011). J'ai pu constater que cette facilitation est particulièrement pertinente pour les élèves jeunes ou en difficulté d'apprentissage (Bétrancourt et Bozelle, 2012). D'autre part, ces interfaces sont en général très appréciées des élèves qui les trouvent plus stimulantes et ludiques, ce qui améliore leur expérience d'utilisation. Des recherches ont bien montré des effets favorables pour des élèves jeunes pour l'apprentissage du tracé des lettres par exemple (Jolly et Gentaz, 2013). Cependant 
cet effet ne se manifeste pas systématiquement. En particulier, une thèse récente (Blanqui, 2020) n'a pas pu démontrer d'effet bénéfique de l'interaction tactile par rapport aux classiques clavier et souris dans l'apprentissage de concepts de physique. Je ferai donc l'hypothèse que pour des apprentissages plus complexes, exigeant de la compréhension conceptuelle, c'est l'adéquation de l'affordance de manipulation avec les processus nécessaires pour comprendre et apprendre qui est importante. Or, la compréhension de concepts nécessite le plus souvent des traitements symboliques, comme le calcul, la lecture et les inférences. Pour revenir à l'idée de congruence technopédagogique mentionnée précédemment et que je soutiens également dans mes travaux, ces interfaces plus directes peuvent soutenir les interactions et les débats dans des situations de type résolution de problème, en particulier dans les moments de mise en commun et de débat, parce qu'elles permettent de réifier les hypothèses des élèves et l'orchestration par l'enseignant, en plus de la facilitation de manipulation. La figure 1 illustre un tel usage dans un projet auquel j'ai participé, où la tablette tactile est utilisée comme moyen de capturer une image de loupe binoculaire et de la partager sur une plateforme dans une activité coopérative en biologie (Widmer et Bétrancourt, 2018).

\section{Figure 1}

Activité de biologie réalisée sur la plateforme Beekee Live (beekee.ch)
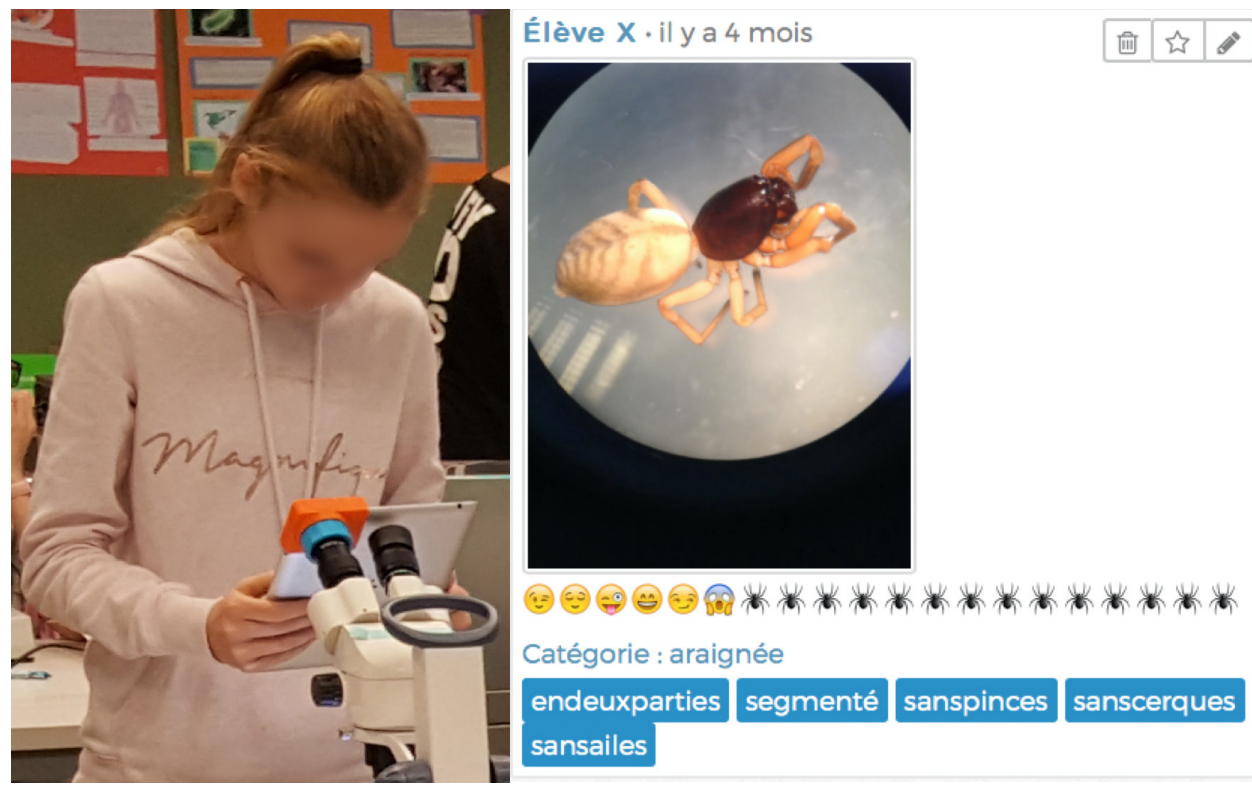

Note. Une élève prend des photos d'un spécimen avec la tablette (à gauche), choisit la meilleure puis la publie sur la plateforme (à droite) pour la suite de l'activité de groupe.

STÉPHANIE FLECK et LUC MASSOU : D'après vos travaux sur les usages des technologies numériques dans différents contextes de formation et d'enseignement, les interactions générées par les environnements numériques modifient-elles, ou non, le rapport aux savoirs scolaires? Quelle vigilance devraient avoir les concepteurs de ces nouveaux systèmes?

MIREILLE BÉTRANCOURT : Pour l'avoir observé lors d'études avec des systèmes tangibles ou des tablettes tactiles, les élèves de primaire et de secondaire souhaitent immédiatement interagir avec ces systèmes même dans des situations scolaires. Leur intérêt pour la matière en est même augmenté si on le mesure par questionnaire. Toutefois, on ne peut exclure ici un effet de nouveauté (Depover, 2009), ces 
outils étant souvent absents des contextes dans lesquels on les a étudiés. D'autre part, dans ces études, les situations pédagogiques sont également nouvelles et stimulantes, en demandant aux élèves de proposer des solutions créatives, de débattre, ce qui n'est pas le quotidien scolaire. Dans l'étude de Widmer et Bétrancourt (2018) illustrée à la figure 1, l'appréciation d'une situation d'apprentissage coopératif en biologie avec des tablettes tactiles était très élevée, alors que les interfaces tactiles sont dans le quotidien de la plupart des élèves de 13 ans depuis leur plus jeune âge. Ainsi est-il possible que ces outils contribuent à montrer aux élèves que les savoirs scolaires ne sont pas déconnectés des autres aspects de leur vie au quotidien. Concernant la vigilance à avoir pour la conception, il me semble important de ne pas privilégier une interaction ludique et stimulante aux dépens de la congruence technopédagogique. On a pu voir cette tentation pour les tables tactiles, dont la plus-value pédagogique en milieu scolaire ne justifiait pas l'investissement, d'autant plus que les interfaces sont développées ad hoc. Par contre, ces tables tactiles ont une grande utilité pour les activités muséales, parce que la manipulation directe facilite l'engagement dans la tâche et favorise l'interaction des visiteurs. Par exemple, une telle table est utilisée très efficacement au Musée international de la Croix-Rouge à Genève dans le cadre d'une simulation coopérative de préparation à l'arrivée d'un ouragan.

STÉPHANIE FLECK et LUC MASSOU : Dans ces recherches, avez-vous observé des changements de posture ou de rôle chez les élèves et/ou chez les enseignants en lien avec leurs usages actuels du numérique?

MIREILLE BÉTRANCOURT : D'après mon expérience, l'une des difficultés de l'usage du numérique en classe est de faire en sorte que la technologie ne reste pas aux mains des enseignants, mais soit aussi aux mains des élèves. Les interfaces dont on parle ici ont toutes cette intention. Pour les enseignants, cela signifie que le contrôle de l'activité est confié aux élèves, ce qui n'est pas toujours facile à gérer pour des questions de gestion de classe, mais également de régulation pédagogique. Les interfaces tangibles ou de réalité augmentée sont encore des systèmes expérimentaux qui, très souvent, sont fermés, au sens où le système contrôle en général assez fortement les actions possibles. Pour les enseignants, le changement de posture est d'accepter une certaine délégation de la gestion de l'activité aux élèves du moins dans certaines phases et, de façon symétrique, que les élèves assument cette responsabilité. Nous avons pendant plusieurs années utilisé un espace wiki dans la formation initiale de professionnels de santé à Genève. Les élèves devaient écrire sur une situation difficile survenue pendant leur stage concernant un thème particulier (par exemple la toilette) et deux autres élèves devaient apporter leurs suggestions de comportement à adopter en les justifiant (Ortoleva et Bétrancourt, 2017). Les enseignantes se sont déclarées surprises de l'engagement des élèves dans cette tâche, alors qu'ils étaient en général réticents à écrire et à participer en situation scolaire. Les élèves peuvent donc assumer cette responsabilité de régulation de l'activité lorsque l'activité pédagogique est engageante, qu'elle fait sens pour les élèves. D'autre part, les enseignants doivent rester en contrôle, c'est-à-dire avoir une visibilité à tout moment de ce que font les élèves, ce qui est facilité par des interfaces tangibles ou des interfaces fournissant un tableau de bord du travail des élèves. Dans le cas de l'étude mentionnée ci-dessus, les enseignantes avaient accès aux pages wiki des élèves pendant l'activité.

STÉPHANIE FLECK et LUC MASSOU : Vous avez été une observatrice privilégiée des mutations qu'ont apportées les interfaces numériques sur l'apprentissage. En particulier, quels sont ou seraient les impacts de ces usages du numérique sur le travail collaboratif entre enseignants et sur leur développement professionnel?

MIREILLE BÉTRANCOURT : II ne faut pas le nier : la mise en place d'activités pédagogiques pertinentes avec des technologies pour certaines encore expérimentales requiert de la part des enseignants un investissement considérable en temps et en formation. Un bon moyen d'alléger le coût de cet investissement est de le partager à plusieurs. Je crois beaucoup à la force des projets collaboratifs d'enseignants ou d'école, plutôt des groupes restreints de personnes qui s'apprécient et partagent les 
mêmes conceptions de l'enseignement que de vastes communautés même si celles-ci ont leur intérêt. Les recherches montrent que l'adoption des technologies numériques est augmentée si ces groupes d'enseignants reçoivent un soutien institutionnel du point de vue des usages des technologies plutôt que d'être livrés à eux-mêmes (Scherer, Siddiq et Tondeur, 2019). Je pense également que les personnes de soutien permettent de faire circuler les bonnes idées entre groupes et établissements.

STÉPHANIE FLECK et LUC MASSOU : Pour conclure, la conception participative des supports technopédagogiques, issue des pays nordiques et portée par les modèles de type fablab schools, est en cours d'expansion dans les pays francophones. Quels avantages et quelles limites éventuelles voyez-vous à ce type d'approche?

MIREILLE BÉTRANCOURT : Nous avons à TECFA des outils de fabrication digitale (découpeusegraveuse de bois, imprimantes $3 \mathrm{D}$, découpeuse plastique, machine à broder). Ces outils permettent en effet aux enseignants de créer leurs propres supports pédagogiques tout en s'appropriant des compétences numériques avancées. En outre, plusieurs portails Web existent pour partager les créations digitales (par exemple : la page Edutechwiki de « Présentation de la conception et fabrication assistées par ordinateur » (n. d.)). Mais comme je le disais précédemment, l'enjeu est que ces technologies ne restent pas aux mains des enseignants, mais soient aussi aux mains des élèves. L'usage pédagogique de ces outils est très intéressant, puisque les élèves ne sont pas seulement utilisateurs de ressources, mais créent leurs propres objets. Selon moi, l'évolution de la société a entrainé les savoirs scolaires vers une surreprésentation des langages symboliques sur la manipulation et la création d'objets concrets. Or, la création d'objets, outre son potentiel de stimuler l'intérêt, développe également de nombreuses compétences, comme la modélisation en trois dimensions et le paramétrage numérique de l'objet.

\section{Liste de références}

Amadieu, F., Mulet, J., Van der Linden, J., Lombard, J. et Van de Leemput, C. (sous presse). Acceptabilité des technologies innovantes: le cas des tablettes. Education Permanente.

Bara, F. et Gentaz, E. (2011). Haptics in handwriting teaching: the role of perceptual and motor skills. Human Movement Science, 30, 745-759.

Bétrancourt, M. (2019). L'ingénierie des dispositifs de e-Formation : une approche psycho-ergonomique. Dans A. Jézégou (dir.), Traité de la eFormation des adultes. Bruxelles: De Boeck.

Bétrancourt, M. et Bozelle, C. (2012). Les MITIC au service de la pédagogie spécialisée : mieux connaître leur spécificité pour développer des usages éducatifs pertinents. Revue suisse de pédagogie spécialisée, 4, 15-23.

Blanqui, D. (2020). Le rôle du geste lors d'une interaction multi-touch sur l'apprentissage et son lien avec les habiletés visuospatiales [thèse de doctorat, Université de Toulouse Jean-Jaures].

Depover, C. (2009). La recherche en technologie éducative : un guide pour découvrir un domaine en émergence. Paris : Éditions des archives contemporaines.

Dillenbourg, P. et Jermann, P. (2010) Technology for Classroom Orchestration. Dans M. Khine et I. Saleh (dir.), New Science of Learning. Springer, New York, NY. https://doi.org/10.1007/978-1-4419-5716-0 26

Goldin-Meadow, S. (2014). How gesture works to change our minds. Trends in Neuroscience and Education, 3(1), 4-6. https://doi.org/10.1016/j.tine.2014.01.002

Hutchins, E., Hollan, J. D. et Norman, D. (1986). Direct manipulation interfaces. Dans S. Draper et D. Norman (dir.), User centred system design (p. 87-124). Lawrence Erlbaum associates.

Jolly, C. et Gentaz, E. (2013). Évaluation des effets d'entraînements avec tablette tactile destinés à favoriser l'écriture de lettres cursives chez des enfants de Cours Préparatoire. STICEF, 20, 1-20. http://sticef.univ-lemans.fr/num/vol2013/02-jolly-atame/sticef 2013 NS jolly 02p.html

Mishra, P. et Keller, M. J. (2006). Technological pedagogical content knowledge: A new framework for teacher knowledge. Teachers College Record, 108(6), 1017-1054. 
Ortoleva, G. et Bétrancourt, M. (2017). L'écriture participative médiatisée comme vecteur du développement professionnel. Raisons éducatives, 21, 108-122.

Présentation de la conception et fabrication assistées par ordinateur (n. d.). Dans Edutechwiki.ch. http://edutechwiki.unige.ch/fmediawiki/index.php?title=Pr\%C3\%A9sentation de la conception et fabrication assi st\%C3\%A9es par ordinateur\&oldid=130690

Rabardel, P. (1995). Les hommes et les technologies, approche cognitive des instruments contemporains. Paris : Armand Colin.

Scherer, R., Siddiq, F. et Tondeur, J. (2019). The technology acceptance model (TAM): A meta-analytic structural equation modeling approach to explaining teachers' adoption of digital technology in education. Computers \& Education, 128(0317), 13-35. https://doi.org/10.1016/j.compedu.2018.09.009

Widmer, V. et Bétrancourt, M. (2018). Les technologies numériques comme soutien à une activité coopérative de travaux pratiques en biologie. RSSE - Revue suisse des sciences de l'éducation, 40(2), 405-446. 